\title{
Variation of mass accretion rate onto the white dwarf in the dwarf nova VW Hyi in quiescence
}

\author{
Nozomi Nakaniwa, ${ }^{1,2 \star}$ Takayuki Hayashi, ${ }^{3}$ Mai Takeo, ${ }^{1,2}$ and Manabu Ishida ${ }^{1,2}$ \\ ${ }_{1}^{1}$ Department of Physics, Tokyo Metropolitan University, 1-1 Minami-Osawa, Hachioji, Tokyo 192-0397, Japan \\ ${ }^{2}$ The Institute of Space and Astronautical Science/JAXA, 3-1-1 Yoshinodai, Chuo-ward, Sagamihara, Kanagawa 252-5210, Japan \\ ${ }^{3}$ NASA's Goddard Space Flight Center, 8800 Greenbelt Road, Greenbelt, MD 20771, USA
}

Accepted 2019 June 29. Received 2019 June 28; in original form 2019 May 17

\begin{abstract}
We have analysed a series of Suzaku data and one dataset of XMM-Newton of the SU UMa type dwarf nova VW Hyi in optical quiescence. The observed spectra in the $0.2-10 \mathrm{keV}$ band are moderately well represented by multi-temperature thermal plasma emission models with a maximum temperature of $5-9 \mathrm{keV}$ and bolometric luminosity of $(2.4-5.2) \times 10^{30} \mathrm{erg} \mathrm{s}^{-1}$. The mass accretion rate derived from the hard X-ray spectra does not show any clear trend as a function of time since the last superoutburst, in contradiction to theoretical predictions of the disc behaviour of a SU UMa type dwarf nova. The mass accretion rate, on the other hand, shows a clear declining trend with time since the last outburst (including the superoutburst). The rate of decline is of the same order as that evaluated from the hard X-ray light curves of the other two dwarf novae SS Cyg and SU UMa. The standard disc instability model, on the other hand, predicts that the mass accretion rate should increase throughout the opticallyquiescent phase. We need further observation and theoretical consideration to resolve this discrepancy.
\end{abstract}

Key words: stars: dwarf novae - novae, cataclysmic variables - X-rays: binaries X-rays: stars.

\section{INTRODUCTION}

A cataclysmic variable (CV) is a binary system composed of a Roche-lobe-filling secondary star and a mass-accreting white dwarf (Warner 2003). The matter spilt over the Roche Lobe forms an accretion disc around the white dwarf. The disc is basically optically thick with an innermost temperature of order $10^{5} \mathrm{~K}$. Hence its radiation appears in the band from infrared to ultraviolet. A dwarf nova (DN) is a subclass of $\mathrm{CV}$ in which an optical outburst occurs with a recurrence time of months to decades. During the outburst, the DN becomes brighter than in quiescence by a few to several magnitudes in the V-band. The accretion disc of the DN is bi-stable. One stable state corresponds to a low accretion-rate state in which the temperature of the outer part of the disc is low (of order $10^{3} \mathrm{~K}$ ) and hydrogen there is neutral. The other is a high accretion-rate state in which the outer part temperature exceeds $10^{4} \mathrm{~K}$ and the hydrogen is ionized. The former and the latter correspond to an optically-quiescent state and an outburst state, respectively. Since the mass accretion rate from the secondary star is intermediate, the DN shows switching behaviour between the

^ E-mail: nakaniwa-nozomi@ed.tmu.ac.jp two states due to thermal instability associated with hydrogen ionization/recombination (Osaki 1974; Hōshi 1979; Meyer \& Meyer-Hofmeister 1981).

Due to lack of any coherent periodic signal, unlike in magnetic cataclysmic variables, it is believed that the magnetic field of the white dwarf is weak, say $B \underset{\sim}{<1} 0^{6} \mathrm{G}$ (van Teeseling et al. 1996), and the accretion disc reaches the white dwarf surface. If the white dwarf rotates much more slowly than the Keplerian velocity at its surface, the accreting matter experiences high friction between the inner accretion disc and the white dwarf surface, and as a result, is abruptly heated. This region is referred to as the boundary layer.

Since the density of the disc is relatively low in the quiescent state, the heating efficiency surpasses the cooling rate in the boundary layer. Consequently, the matter in the boundary layer swells, leaves the orbital plane, and forms an optically thin thermal plasma with a temperature of order $10^{8} \mathrm{~K}$, from which hard X-ray emission emanates. In the outburst state, on the other hand, the cooling efficiency is in general high enough to keep the disc optically thick, and the emission from the disc extends to EUVE region (Pringle \& Savonije 1979; Patterson \& Raymond 1985). The transition between these two states was clearly detected 
with the multi-waveband observation of SS Cygni by Wheatley et al. (2003), in which an outburst in the EUVE band was delayed from that in optical by $\sim 1 \mathrm{~d}$, and its onset coincided with an abrupt decline of the hard X-ray emission in the $2-15 \mathrm{keV}$ band.

The hot plasma in the boundary layer of the quiescent state is formed by the frictional heating of the disc matter, is cooled via optically thin thermal plasma emission, and is finally settled onto the white dwarf surface. Hence it is multi-temperature. As a matter of fact, a cooling flow model that was originally developed for clusters of galaxies (Mushotzky \& Szymkowiak 1988; Fabian 1994) and its slightly modified version has been successful in fitting DN hard X-ray spectra in general (Baskill et al. 2005; Pandel et al. 2005; Wada et al. 2017).

VW Hydri is a DN whose primary and secondary masses are $0.63 \pm 0.15 M_{\odot}$ and $0.11 \pm 0.02 M_{\odot}$, respectively, and the orbital period is $107.0 \mathrm{~min}$ (Schoembs \& Vogt 1981; Ritter \& Kolb 2003). There are a couple of estimations of the distance to VW Hyi: 65 pc (Warner 1987) and $82 \pm 5$ pc (Barrett 1996). Recently, however, the Gaia collaboration (Gaia Collaboration et al. 2016) published results of their highly accurate parallax measurements. Just inverting the parallax of VW Hyi from data release 2 (DR2, Gaia Collaboration et al. 2018), we obtained the distance to VW Hyi as $54 \pm 0.1$ pc.

As can be recognized from the orbital period, VW Hyi is an SU UMa-type DN which shows not only the normal outburst but also a superoutburst. The superoutburst is brighter in the optical band than the normal outburst by $\sim 1$ mag at its peak, and lasts for $\sim 7-10 \mathrm{~d}$, longer than the normal outburst ( a few days). During the normal outburst, the disc mass continues to decline due to accretion onto the white dwarf, but the total mass loss per outburst is smaller than the gain of mass between the two outbursts. Hence the disc gradually gets heavier and extends outward. Once the disc grows large enough, a thermal-tidal instability occurs, triggered by a resonance between the orbital motion and the Keplerian motion of the disc, leading to the superoutburst (Whitehurst 1988; Osaki 1989; Hirose \& Osaki 1990; Osaki 1996). In the case of VW Hyi, the normal outburst and the superoutburst occur every $\sim 30 \mathrm{~d}$ and $\sim 180 \mathrm{~d}$, respectively (Ritter \& Kolb 2003).

The X-ray behaviour of VW Hyi has been investigated using various observatories. Wheatley et al. (1996) observed a termination phase of an outburst in the 2$10 \mathrm{keV}$ band with Ginga, and fitted the cooling flow model (Mushotzky \& Szymkowiak 1988) to the combined Ginga and ROSAT spectra. This gave a maximum temperature of the boundary layer of $11_{-2}^{+3} \mathrm{keV}$. The flux in the $0.04-10 \mathrm{keV}$ band was $2.08 \times 10^{-11} \mathrm{erg} \mathrm{cm}^{-2} \mathrm{~s}^{-1}$. Pandel et al. (2003) observed VW Hyi with XMM-Newton during the middle of a normal outburst and the following superoutburst. The cooling flow model results in $T_{\max }=7.8 \pm 0.2 \mathrm{keV}$ with a bolometric flux of $7.8 \times 10^{-12} \mathrm{erg} \mathrm{cm}^{-2} \mathrm{~s}^{-1}$. Baskill et al. (2005) analysed a couple of $A S C A$ datasets of VW Hyi in the quiescent state. One was taken at the middle of the end of a superoutburst and the following normal outburst, and the other is $\sim 17 \mathrm{~d}$ after a normal outburst. The intensity of the former observation is $\sim 4$ times as large as the latter. The flux of the former in the band $0.8-10 \mathrm{keV}$ was $4.7 \times 10^{-12}$ erg $\mathrm{cm}^{-2} \mathrm{~s}^{-1}$ with a fixed $T_{\max }$ of $20 \mathrm{keV}$.

In this paper, we present results from a series of VW Hyi observations carried out with Suzaku in the opticallyquiescent state. There is a prediction of the time evolution of the mass transfer rate through the outer disc within a supercycle. The mass transfer rate suddenly increases at the onset of a normal/super outburst and continues to decline to the end of the outburst. During the interval period of two neighbouring outbursts, the transfer rate is predicted to increase by degrees due to accumulation of mass from the secondary (Osaki 1996, and references therein). On the other hand, there has been no systematic study of the mass transfer rate in the boundary layer in relation to the supercycle phase. Since the boundary layer in the quiescent state mainly emits hard X-rays, we have decided to analyse Suzaku data systematically to elucidate the mass accretion history in the quiescent state. This paper is organized as follows. In $\S 2$, we summarize the Suzaku observations of VW Hyi in the quiescent state. Observation windows and data selection criteria are described. We also reanalysed the XMM-Newton data of VW Hyi in quiescence, which is already published by Pandel et al. (2003). In $\S 3$, we explain our spectral analysis in detail. $\S 4$ is devoted to discussion. We primarily concentrate on the mass accretion rate history in relation to the supercycle or the outburst cycle. Finally, we summarize our results in $\S 5$.

\section{OBSERVATIONS AND DATA REDUCTION}

\subsection{Suzaku Observation}

Suzaku (Mitsuda et al. 2007) observed VW Hyi in the quiescent state three times in 2011-2012. Suzaku is equipped with two kinds of detector systems. One is the X-ray Imaging Spectrometer (XIS; Koyama et al. 2007), which adopts a Charge-Coupled Device (CCD). Of the four modules XIS0 through XIS3, XIS1 is a back-illuminated (BI) CCD whereas the others are front-illuminated (FI) CCDs. Each XIS module is mounted in the focal plane of the corresponding X-Ray Telescope (XRT; Serlemitsos et al. 2007) whose focal length is $4.75 \mathrm{~m}$. Note, however, that XIS2 has not been functional since 2006 November 9th, probably because of a micrometeorite collision. The other detector system is the Hard Xray Detector (HXD; Takahashi et al. 2007; Kokubun et al. 2007), which is a non-focusing hard X-ray detector covering 10-600 keV. We do not use the HXD data, because VW Hyi was too faint for spectral analysis in the HXD band. Table 1 summarizes the Suzaku observations of VW Hyi in the quiescent state. In Fig. 1 the Suzaku observation windows in the quiescent state are shown overlaid on the optical light curve taken from the homepage of the American Association of Variable Star Observers (AAVSO) ${ }^{1}$. In addition to these quiescence data, there is one more dataset taken near the peak of the superoutburst (around day 60 in Fig. 1). The observation and the analysis results are summarized in Wada et al. (2017), together with those from quiescence.

\footnotetext{
1 https://www.aavso.org
} 
Table 1. Suzaku observation log of VW Hyi with Suzaku and XMM-Newton.

\begin{tabular}{|c|c|c|c|c|c|c|}
\hline Sequence\# & $\begin{array}{c}\text { Observation date } \\
\text { (UT) }\end{array}$ & Observatory & Detector & Mode & $\begin{array}{l}\text { Exposure }^{\dagger} \\
(\mathrm{ks})\end{array}$ & $\begin{array}{l}\text { Intensity } \\
\left(\text { count s } ~^{-1}\right)^{\S}\end{array}$ \\
\hline \multirow[t]{2}{*}{4006009020} & 2011 Dec 29 15:19:16 - 04:20:11 & Suzaku & XIS FI & Normal & 18.35 & $1.082 \pm 0.008$ \\
\hline & & & XIS BI & Normal & 18.35 & $0.794 \pm 0.007$ \\
\hline \multirow{2}{*}{4006009030} & 2012 Feb 29 13:39:04 - 06:00:11 & Suzaku & XIS FI & Normal & 22.83 & $0.928 \pm 0.007$ \\
\hline & & & XIS BI & Normal & 22.83 & $0.633 \pm 0.006$ \\
\hline \multirow[t]{2}{*}{4006009040} & 2012 May 02 19:29:54 - 04:00:13 & Suzaku & XIS FI & Normal & 18.08 & $0.994 \pm 0.008$ \\
\hline & & & XIS BI & Normal & 18.08 & $0.654 \pm 0.006$ \\
\hline \multirow[t]{3}{*}{0111970301} & 2001 Oct 19 05:31:04 - 10:42:58 & $X M M-N e w t o n$ & $\operatorname{MOS} 1$ & Normal & 18.17 & $0.656 \pm 0.006$ \\
\hline & & & MOS 2 & Normal & 18.17 & $0.688 \pm 0.007$ \\
\hline & & & PIN & Normal & 14.45 & $2.354 \pm 0.013$ \\
\hline
\end{tabular}

After data screening which is described in $\S 2.3$.

* In the band 0.5-10.0 keV for the XIS FI (XIS0 + XIS3) and 0.3-10.0 keV for the XIS BI.

$\S$ After background subtraction. The source and background regions are explained in $\S 3.1$.

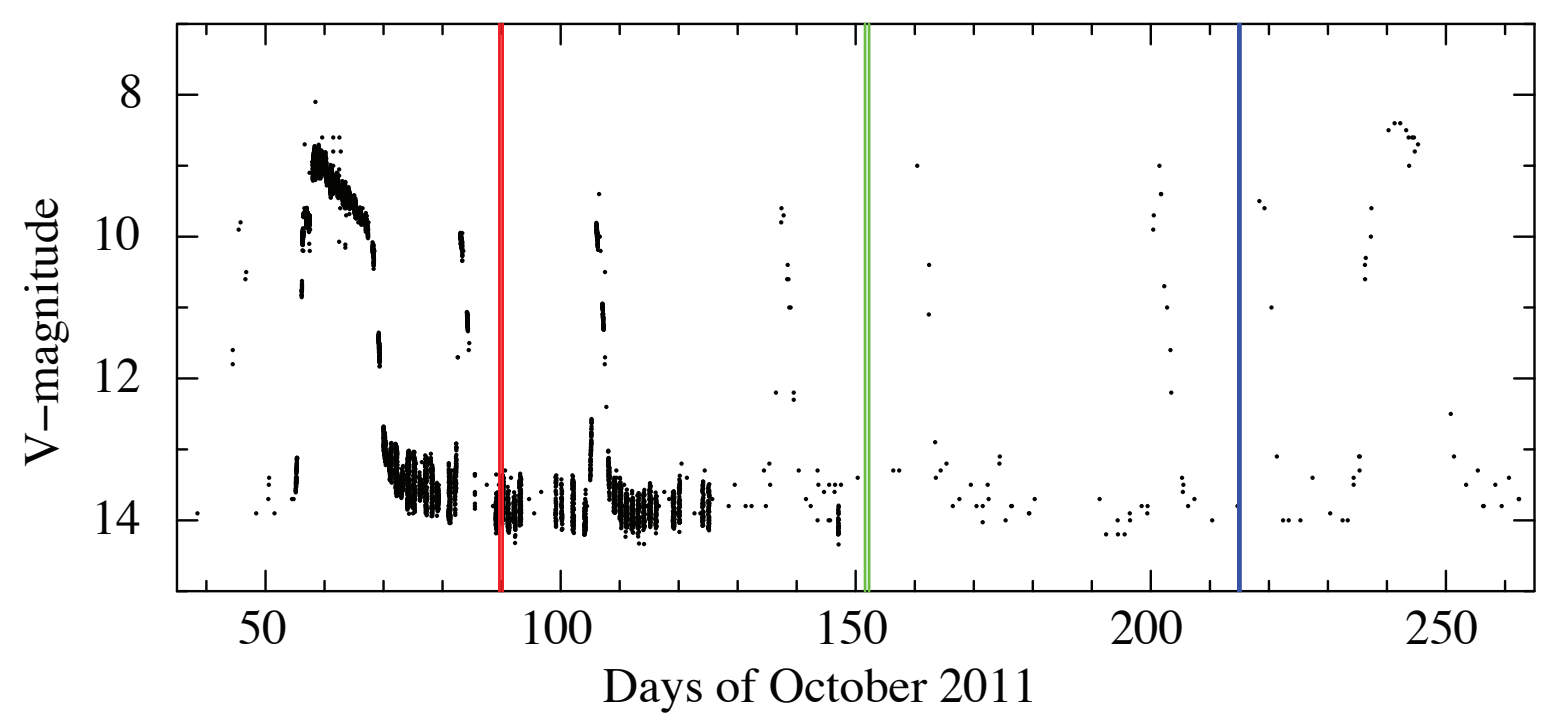

Figure 1. The Suzaku observation windows (the red, green and blue bands) overlaid on the optical light curve, which is taken from the AAVSO homepage. The abscissa shows the elapsed time in days since 2011 October 1st 0:00:00 (UT).

\subsection{XMM-Newton Observation}

As well as Suzaku, XMM-Newton (Jansen et al. 2001) has observed VW Hyi in its quiescent state, which has already been published by Pandel et al. (2003). Since the effective area of XMM-Newton is large and advantageous for spectroscopy of a faint source like VW Hyi, we have decided to incorporate these data in our analysis. The observation log of $X M M-N e w t o n$ is also listed in table 1 , and the observation epoch is shown overlaid on the AAVSO light curve in Fig. 2. The observation was carried out during an epoch between a normal outburst and a following superoutburst in 2001.

\subsection{Data selection criteria}

\subsubsection{Suzaku}

We have screened the Suzaku data with XSELECT version $2.4 \mathrm{e}$, which is included in the HEASOFT package provided by NASA's Goddard Space Flight Center (GSFC) ${ }^{2}$. We have mostly followed the standard data selection criteria. We have only used data while the telemetry rate is either high or medium. We have removed bad pixels and flickering pixels, and have selected events of grade $0,2,3,4$ and 6 . We have discarded data taken during spacecraft passages through the South Atlantic Anomaly and when the pointing accuracy is low. Also discarded are data taken while the elevation of VW Hyi from the night earth limb is less than $5 \mathrm{deg}$. On the other hand, the standard data screening criteria recommend not to use the data when the target star is within $20 \mathrm{deg}$ from the bright earth limb. The number, however, depends upon attitude and orbit condition at the time of the observation. In addition to this, VW Hyi lies close to the south ecliptic pole, and hence the XIS field of view tends to be close to the earth limb. These facts suggest that significant amounts of data would be discarded with the standard day-earth elevation angle. Accordingly, we have drawn spectra with various

\footnotetext{
2 https://heasarc.gsfc.nasa.gov/docs/software.html
} 


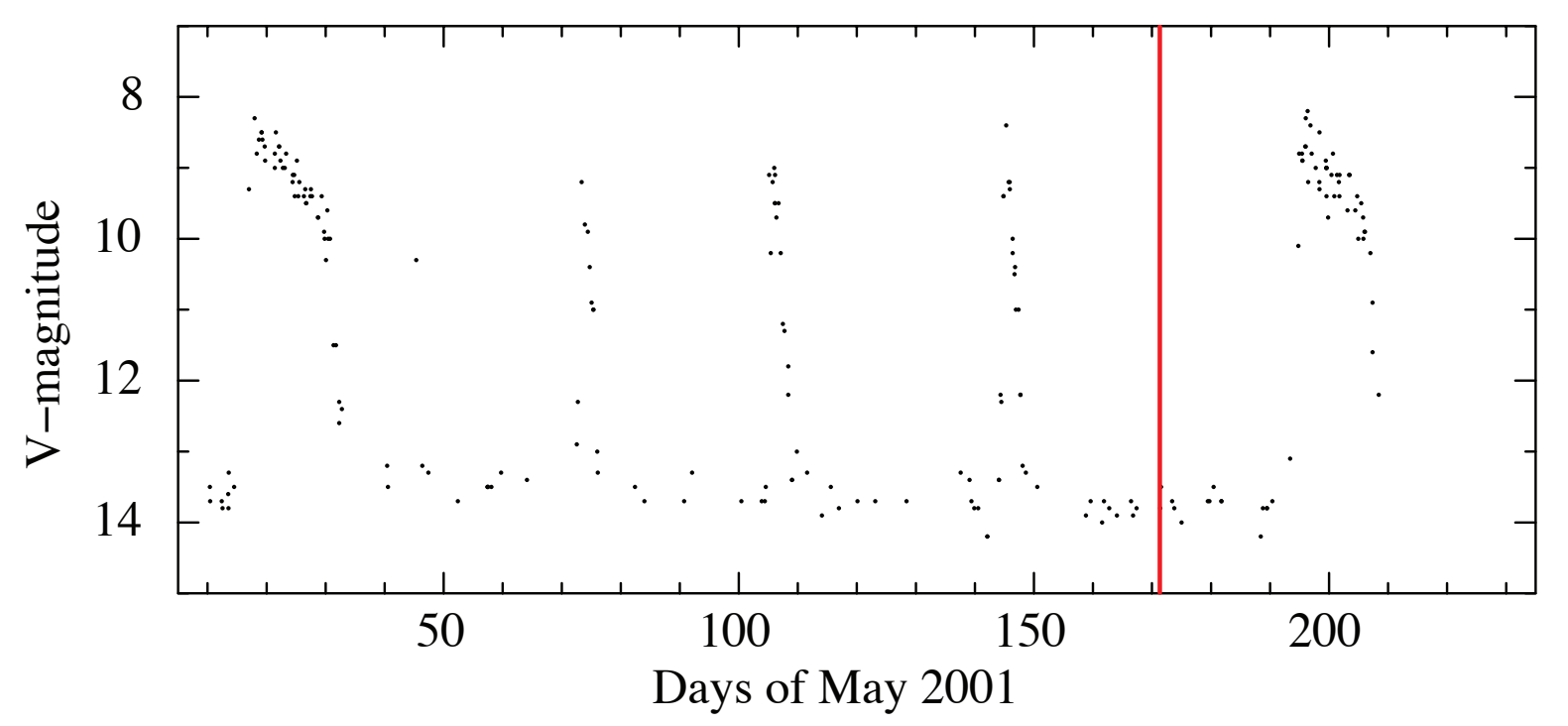

Figure 2. The XMM-Newton observation window (the red band) overlaid on the optical light curve, which is taken from AAVSO homepage. The abscissa shows the elapsed time in days since 2001 May 1st 0:00:00 (UT).

trial day-earth elevation angles and, by closely inspecting the spectra in the band $0.3-0.7 \mathrm{keV}$, where the atmospheric Nitrogen and Oxygen lines appear, we have found that the cutoff day-earth elevation angle can be set as small as $7 \mathrm{deg}$. The exposure times listed in table 1 are those after applying the new day-earth elevation threshold. As we expected, the exposure times are increased by $7-14 \%$.

\subsubsection{XMM-Newton}

To increase observation coverage over the supercycle phase, we reanalysed the XMM-Newton data of VW Hyi, which were published by Pandel et al. (2003). We have used the Science Analysis Software (SAS) XMMSAS_20160201_183315.0.0. We have followed the standard data selection criteria.

\section{ANALYSIS AND RESULTS}

\subsection{Extraction of spectra}

In extracting spectra of the Suzaku XIS, we have collected $\mathrm{X}$-ray photons arriving in a circular aperture with a radius of 3'.5 centered on VW Hyi. The background photons have been integrated over an annular region with inner and outer radii of $4^{\prime}$ and 6.5, respectively. As for the XMM-Newton MOS and pn, the source photons have been accumulated with a circular aperture of 40 " radius, while those of the background are from an annulus with inner and outer radii of $40^{\prime \prime}$ and $60^{\prime \prime}$, respectively.

The spectra thus obtained are shown in Fig. 3. The insets are blow-ups of the iron $\mathrm{K} \alpha$ emission line energy band. Although statistics are somewhat limited, the spectra are characterized by He-like and hydrogenic $\mathrm{K} \alpha$ emission lines (hereafter referred to as $\mathrm{He} \alpha$ and $\mathrm{Ly} \alpha$, respectively) of $\mathrm{Mg}$ (1.34 keV and $1.47 \mathrm{keV}), \mathrm{Si}(1.86 \mathrm{keV}$ and $2.01 \mathrm{keV}), \mathrm{S}$ (2.45 keV and $2.62 \mathrm{keV})$ and $\mathrm{Fe}(6.68 \mathrm{keV}$ and $6.96 \mathrm{keV})$. This fact strongly suggests that the X-ray emission from
VW Hyi originates from a thermal plasma with multiple temperatures. As recognized from the insets, the iron $\mathrm{K} \alpha$ emission is dominated by the $\operatorname{He} \alpha$ component, whereas $\operatorname{Ly} \alpha$ is detected only marginally. This implies that the maximum temperature of the plasma is lower than $\sim 10 \mathrm{keV}$. It is also remarkable from the insets that the neutral iron $\mathrm{K} \alpha$ line $(6.40 \mathrm{keV})$ seems absent. This is a big contrast to the archetypal DN SS Cygni (Ishida et al. 2009).

\subsection{Response functions}

In preparation for spectral analysis, we have made redistribution matrix files (RMFs) of the Suzaku XIS with the software XISRMFGEN (version 2012-04-21). With the RMF thus made, we have then created ancillary response files (ARFs) with XISSIMARFGEN (version 2010-11-05). The information of the photon-integration region is taken into account here. The RMFs and the ARFs thus produced are merged with MARFRMF (v3.2.6) for the three XIS modules separately, and the resultant response matrix files of the XIS0 and XIS3 (FI CCDs) are coadded with ADDRMF.

We have followed a similar procedure in preparing the response matrix files for XMM-Newton MOS and pn. We have made the RMFs of MOS1, MOS2, and pn separately with RMFGEN (version 2.2.1) and the ARFs with ARFGEN (version 1.92), which are both included in the package xmmsas_20160201_1833-15.0.0.

\subsection{Spectral models}

In evaluating the spectra, we use the software XSPEC (Arnaud 1996) version 12.10.0 in the HEASOFT package provided by NASA's GSFC ${ }^{3}$. Based upon the appearance of the spectra described above, as well as upon accumulated knowledge of the DNe X-ray spectra, we here adopt the following models. Throughout this paper, we adopt

3 http://heasarc.gsfc.nasa.gov 


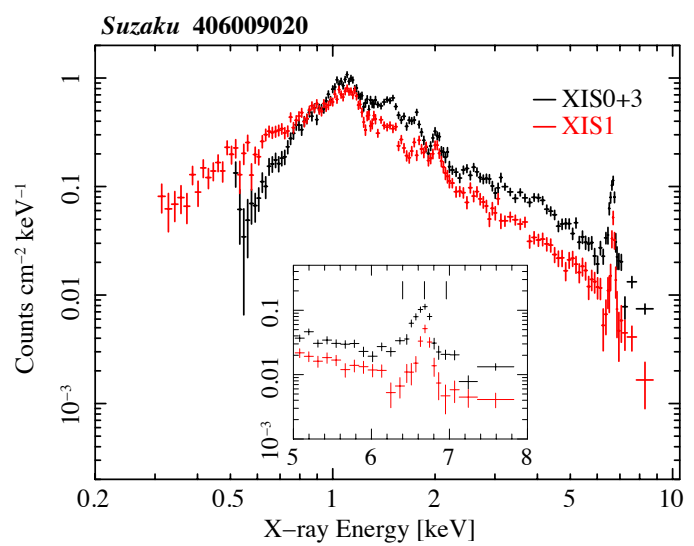

Suzaku 406009030

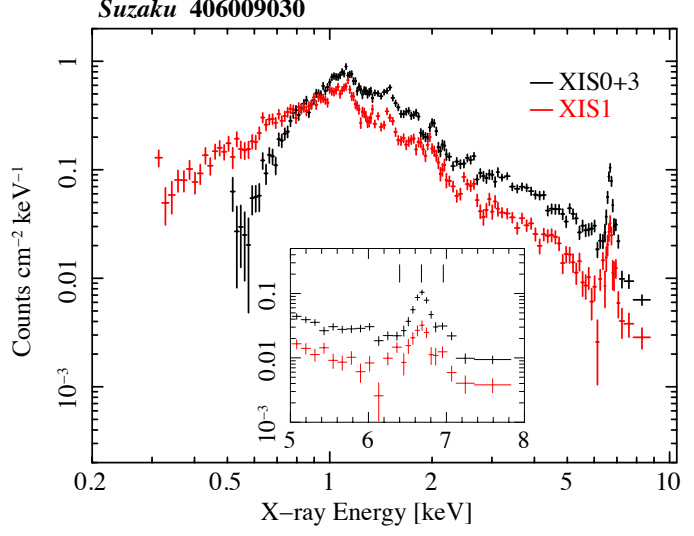

Suzaku 406009040

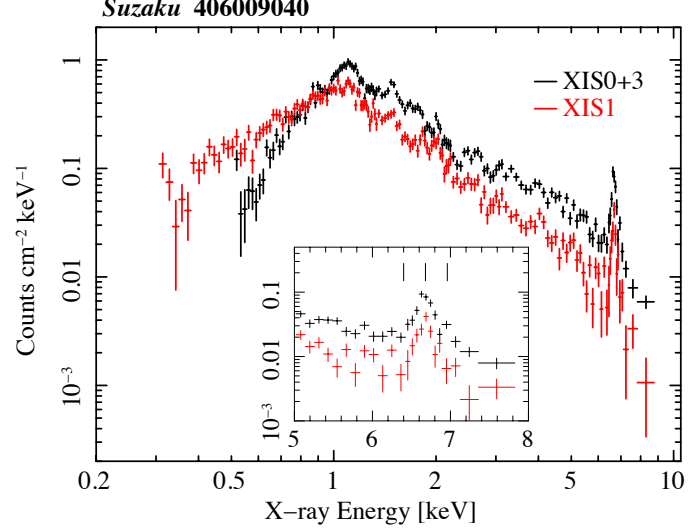

XMM-Newton 0111970301

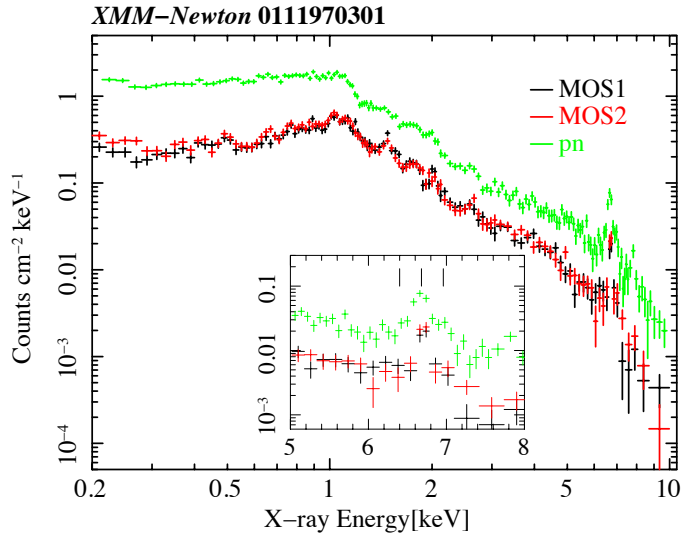

Figure 3. VW Hyi spectra of Suzaku (seq. \#406009020, 406009030, 406009040) and XMM-Newton (seq. \#0111979301). The tick marks of the insets indicate the energies of iron $\mathrm{K} \alpha$ emission lines from neutral, He-like, and hydrogenic ionization states. Note that the ordinate range of the XMM-Newton main frame is different from that of Suzaku.
$N_{\mathrm{H}}=6 \times 10^{17} \mathrm{~cm}^{-2}$ (Polidan et al. 1990) as the interstellar absorption hydrogen column density to VW Hyi.

\subsubsection{Multiple temperature plasma emission models}

We have adopted the following two spectral models to represent the observed multiple temperature plasma emission. One is the VMCFLOW model. As can be deduced from its name, this model is originally developed to model X-ray spectra of a cooling flow that was presumed to appear in galaxy clusters (Mushotzky \& Szymkowiak 1988; Fabian 1994). Probably because the heating and cooling processes that take place in the boundary layer is similar to those in the clusters of galaxies, VMCFLOW has been found to succeed in representing X-ray spectra of DNe in general (Pandel et al. 2005; Baskill et al. 2005; Wada et al. 2017). In this model, the mass accretion rate $\dot{M}$ is one of the model parameters which is evaluated from the equation

$L_{\text {bol }}=\frac{5}{2} \frac{\dot{M}}{\mu m_{\mathrm{H}}} k_{\mathrm{B}} T_{\max }$,

where $T_{\max }$ is the maximum temperature of the plasma, which is again one of the model parameters, and $L_{\mathrm{bol}}$ is the bolometric luminosity of the source, which is evaluated accurately, given the distance to VW Hyi of 54 pc $(\S 1)$.

The other multiple temperature plasma model is CEVMKL. In VMCFLOW, the temperature dependence of the differential emission measure $(D E M)$ is subject to the temperature dependence of the volume emissivity of the plasma (so called "cooling function"; see Gehrels \& Williams 1993, for example). In CEVMKL, on the other hand, the DEM is replaced with a power-law function of the temperature.

$d(E M) \propto\left(\frac{T}{T_{\max }}\right)^{\alpha} d(\log T) \propto\left(\frac{T}{T_{\max }}\right)^{\alpha-1} d T$ for $T<T_{\max }$

\subsubsection{Reflection model}

Since the boundary layer plasma is formed in contact with the white dwarf surface, a significant fraction of its X-ray emission is intercepted by the white dwarf and a part of it is reflected off into space. The height of the boundary layer from the white dwarf surface has been estimated using the eclipse by the secondary star in the SU UMa-type DN HT Cas (Mukai et al. 1997) to be $<0.15 R_{\mathrm{WD}}$ where $R_{\mathrm{WD}}$ is the radius of the white dwarf, and using the intensity of the iron $\mathrm{K} \alpha$ emission line to be $<0.12 R_{\mathrm{WD}}$ in SS Cyg (Ishida et al. 2009). In the latter case, a strong fluorescence $\mathrm{K} \alpha$ line from neutral iron at $6.40 \mathrm{keV}$ is detected with an equivalent width of $\simeq 100 \mathrm{eV}$. Although such a strong line does not seem to be detected from VW Hyi, we have added a plasma emission component that is reflected off the white dwarf to estimate the intensity of the reflected component. For this, we adopt the XSPEC model REFLECT (Magdziarz \& Zdziarski 1995).

\subsection{Evaluation of the spectra}

We have attempted to fit the VMCFLOW and CEVMKL models to the Suzaku and XMM-Newton spectra in quiescence. 
Table 2. Best-fit continuum parameters with the vMCFLOW model

\begin{tabular}{lcccc}
\hline Seq. \# & 406009020 & 406009030 & 406009040 & 0111970301 \\
\hline$N_{\mathrm{H}}\left(10^{17} \mathrm{~cm}^{-2}\right)$ & & $6.0^{\dagger}$ (fixed) & \\
$\Omega / 2 \pi$ & & $1.5^{\ddagger}$ (fixed) & \\
$i$ & & $60^{\circ}$ (fixed) & \\
$T_{\max }(\mathrm{keV})$ & $8.61_{-0.26}^{+0.17}$ & $8.86_{-0.22}^{+0.31}$ & $8.67_{-0.22}^{+0.20}$ & $6.95_{-0.19}^{+0.20}$ \\
$\dot{M}\left(10^{-12} M_{\odot} \mathrm{yr}^{-1}\right)$ & $2.45_{-0.05}^{+0.07}$ & $1.92_{-0.06}^{+0.05}$ & $2.02_{-0.05}^{+0.05}$ & $1.40_{-0.03}^{+0.03}$ \\
\hline Flux $^{\S \S}\left(10^{-12} \mathrm{erg} \mathrm{cm}^{-2} \mathrm{~s}^{-1}\right)$ \\
$0.2-12 \mathrm{keV}$ & 12.63 & 10.18 & 10.49 & 5.81 \\
Bolometric & 14.95 & 12.07 & 12.41 & 6.81 \\
\hline Luminosity ${ }^{\S}\left(10^{30} \mathrm{erg} \mathrm{s}^{-1}\right)$ \\
\multicolumn{5}{c}{ Bolometric } \\
\hline$\chi^{2}$ (d.o.f.) & 5.21 & 4.21 & 4.33 \\
\hline
\end{tabular}

Note: All errors are single parameter 90\% confidence level.

$\dagger$ Hydrogen column density to VW Hyi (Polidan et al. 1990).

$\ddagger$ Solid angle of cold matter surrounding the boundary layer

(Ishida et al. 2009).

$\S$ Inclination angle of the reflector (primarily accretion disc), which is regarded as identical with the orbital inclination angle (Ritter \& Kolb 2003).

$\S \S$ The reflected component being excluded $(\Omega$ is set equal to zero after the fit converged).

|l Flux or luminosity in the band $0.04-100 \mathrm{keV}$.

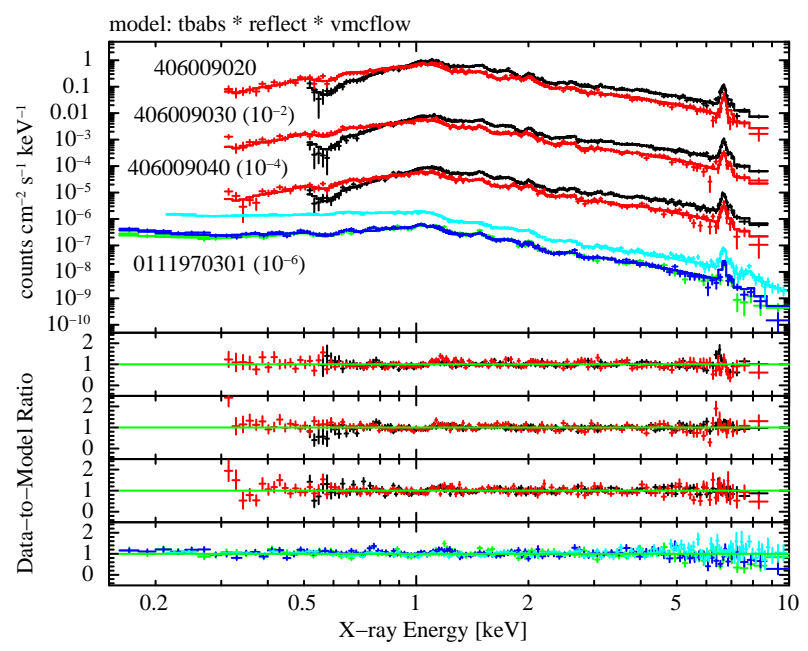

Figure 4. Simultaneous fit of the VMCFLOW model to the Suzaku and $X M M-N e w t o n$ spectra. Interstellar absorption and the reflection are considered with the models TBABS $\left(\left(N_{\mathrm{H}}\right.\right.$ fixed at $6 \times 10^{17} \mathrm{~cm}^{-2}$; see Polidan et al. 1990) and REFLECT.

These models include abundances of the major metals as parameters. In order to make them common among all the spectra, we have tried to fit the four sets of data (three from Suzaku and one from XMM-Newton) simultaneously with the metal abundances being set free to vary but constrained to have common values. The best-fit continuum parameters with the VMCFLOW and CEVMKL models are summarized in table 2 and 3, respectively. The resultant elemental abundances with these models are listed in table 4 . The result of the fit with the VMCFLOW model is shown in Fig. 4.

From the $\chi^{2}$ values, the CEVMKL model fits better to the observed spectra than the VMCFLOW model. The CEVMKL
Table 3. Best-fit continuum parameters with the CEVMKL model

\begin{tabular}{|c|c|c|c|c|}
\hline Seq. \# & 406009020 & 406009030 & 406009040 & 0111970301 \\
\hline$N_{\mathrm{H}}\left(10^{17} \mathrm{~cm}^{-2}\right)$ & & $6.0^{\dagger}$ & (fixed) & \\
\hline$\Omega / 2 \pi$ & & $1.5^{\ddagger}$ & (fixed) & \\
\hline$i$ & & $60^{\circ \S}$ & (fixed) & \\
\hline$T_{\max }(\mathrm{keV})$ & $5.06_{-0.23}^{+0.24}$ & $5.88_{-0.17}^{+0.39}$ & $5.77_{-0.38}^{+0.16}$ & $5.32_{-0.22}^{+0.23}$ \\
\hline$\alpha$ & $1.90_{-0.11}^{+0.12}$ & $1.59_{-0.10}^{+0.07}$ & $1.58_{-0.06}^{+0.16}$ & $1.45_{-0.07}^{+0.07}$ \\
\hline \multicolumn{5}{|c|}{ Flux $^{\S \S}\left(10^{-12} \mathrm{erg} \mathrm{cm}^{-2} \mathrm{~s}^{-1}\right)$} \\
\hline $0.2-12 \mathrm{keV}$ & 12.36 & 10.07 & 10.35 & 5.76 \\
\hline Bolometric" & 14.16 & 11.69 & 12.01 & 6.78 \\
\hline \multicolumn{5}{|c|}{ Luminosity $^{\S}\left(10^{30} \mathrm{erg} \mathrm{s}^{-1}\right)$} \\
\hline Bolometric" & 4.94 & 4.07 & 4.19 & 2.36 \\
\hline$\chi^{2}$ (d.o.f.) & \multicolumn{4}{|c|}{$1623.98(1283)$} \\
\hline
\end{tabular}

Note: All errors are single parameter $90 \%$ confidence level.

$\dagger$ Hydrogen column density to VW Hyi (Polidan et al. 1990).

\$ Solid angle of cold matter surrounding the boundary layer

(Ishida et al. 2009).

$\S$ Inclination angle of the reflector (primarily accretion disc), which is regarded as identical with the orbital inclination angle (Ritter \& Kolb 2003).

$\S \S$ The reflected component being excluded ( $\Omega$ is set equal to zero after the fit converged).

II Flux or luminosity in the band $0.04-100 \mathrm{keV}$.

Table 4. Best-fit elemental abundances with the VMCFLOW and CEVMKL models

\begin{tabular}{ccc}
\hline & VMCFLOW & CEVMKL \\
\hline $\mathrm{O}$ & $1.02_{-0.10}^{+0.10}$ & $0.93_{-0.10}^{+0.10}$ \\
$\mathrm{Mg}$ & $1.56_{-0.18}^{+0.18}$ & $1.02_{-0.18}^{+0.19}$ \\
$\mathrm{Si}$ & $1.53_{-0.13}^{+0.13}$ & $1.18_{-0.13}^{+0.13}$ \\
$\mathrm{~S}$ & $1.52_{-0.19}^{+0.19}$ & $1.12_{-0.17}^{+0.17}$ \\
$\mathrm{Ar}$ & $1.71_{-0.53}^{+0.53}$ & $1.14_{-0.45}^{+0.46}$ \\
$\mathrm{Ca}$ & $1.63_{-0.67}^{+0.66}$ & $1.35_{-0.55}^{+0.55}$ \\
$\mathrm{Fe}$ & $0.95_{-0.04}^{+0.84}$ & $0.90_{-0.04}^{+0.84}$ \\
$\mathrm{Ni}$ & $2.49_{-0.52}^{+0.53}$ & $1.92_{-0.49}^{+0.50}$ \\
\hline
\end{tabular}

Note - Elemental abundances relative to those of the sun (Anders \& Grevesse 1989) are tabulated. Abundances of $\mathrm{He}, \mathrm{C}, \mathrm{N}, \mathrm{Ne}, \mathrm{Na}$ and $\mathrm{Al}$ are fixed to unity.

model has four additional free parameters compared with VMCFLOW model. An $F$-test reveals, however, that the addition of the DEM slope parameter ( $\alpha$ in eq.(2)) is significant at more than $99 \%$ confidence level. The advantage of the CEVMKL model over the VMCVLOW model is also suggested by Pandel et al. (2005) from comprehensive analysis of DNe data by XMM-Newton.

The solid angle $\Omega / 2 \pi$ of the reflector subtending over the boundary layer plasma is fixed at 1.5. This is based on the result of the spectral evaluation of SS Cyg in quiescence $(\Omega / 2 \pi=1.7 \pm 0.2$; see Ishida et al. 2009). As mentioned in $\S 3.3 .2$, the spatial extent of the boundary layer is as small as $\sim 0.1 R_{\mathrm{WD}}$. If the boundary layer is small enough compared with the white dwarf, the surface of the white dwarf is viewed from the plasma as an infinite plane covering half of the sky $(\Omega / 2 \pi=1)$. The accretion disc is further thought to cover half of the remaining sky $(\Omega / 2 \pi=0.5)$. In reality, the solid angle should be less than 1.5 , because the boundary 
layer has a finite size. Nevertheless, the fitting is insensitive to the value of $\Omega / 2 \pi$, because the reflection component occupies only a small fraction of the entire spectrum, as will be explained in $\S 4.2$ (see Fig. 5). For the same reason, we have fixed the inclination of the reflector at the same value as the orbital one $60^{\circ}$ (Ritter \& Kolb 2003).

In the case of using the CEVMKL model, the maximum temperature of the plasma is nearly constant, being confined in the narrow range 5-6 keV (table 3). The DEM slope $\alpha$ of the first Suzaku observation (seq. \#40609020) is, on the other hand, slightly larger than the other three observations. This means that the spectrum of the first Suzaku observation is slightly harder than the others. Based on the similarity of $T_{\max }$ and $\alpha$, the spectral shapes of the other three observations resemble one another, although the flux of the $X M M$ Newton observation (seq. \#0111970301) is roughly half of the second and third Suzaku observations (seq. \#406009030 and \#406009040). Note that the flux difference between the Suzaku and XMM-Newton detectors is in general less than 10\% (Tsujimoto et al. 2011; Ishida et al. 2011). Hence, the smaller flux obtained with XMM-Newton by a factor of two is significant. The resultant abundances from the VMCFLOW model are in general greater than those from the CEVMKL model. This is because $T_{\max }$ is larger in the VMCFLOW model; a higher temperature in the $\mathrm{keV}$ range reduces the volume emissivity of the $\mathrm{K} \alpha$ emission lines from abundant elements in He-like and hydrogenic ionization states, and hence requires a larger abundance to explain the observed intensities of those emission lines. The spectral fits with the CEVMKL model result in abundances fairly close to solar values (table 4), which is consistent with the results of Pandel et al. (2005).

\subsection{Evaluation of mass accretion rate}

As explained in $\S 3.4$, the model VMCFLOW includes the mass accretion rate as one of its free parameters. Its best-fit values are listed in table 5 (the same as in table 2 ). In order to confirm these mass accretion rates, as well as to estimate their systematic errors, we have attempted to calculate them from the observed bolometric fluxes which are listed in tables 2 and 3 for the VMCFLOW and CEVMKL models, respectively. For the conversion to the mass accretion rate, we need the mass and the radius of the white dwarf. As described in $\S 1$, the mass of the white dwarf has been estimated as $M_{\mathrm{WD}}=0.63 \pm 0.15 M_{\odot}$ (Schoembs \& Vogt 1981). With this mass, the radius can be obtained from the mass-radius relation of Nauenberg $(1972)$ as $R_{\mathrm{WD}}=\left(8.4_{-1.2}^{+1.5}\right) \times 10^{8} \mathrm{~cm}$. As a result, the mass accretion rate can be calculated with the following equation.

$4 \pi D^{2} f_{\mathrm{bol}}=\frac{1}{2} \frac{G M_{\mathrm{WD}} \dot{M}}{R_{\mathrm{WD}}}$,

where $D=54 \mathrm{pc}$ is the distance to the source $(\S 1), f_{\mathrm{bol}}$ is the bolometric flux listed in table 2 and 3 . The factor $1 / 2$ on the right-hand side reflects the fact that half of the accretion energy is lost in the disc before the accreting matter enters the boundary layer.

The mass accretion rates thus obtained are listed in table 5. From this table, they are found to be smaller than the best-fit values of the VMcFlow model in general. Some possible reasons for this will be discussed in $\S 4.3$.

\section{DISCUSSION}

\subsection{Continuum model}

As described in $§ 3.4$, the CEVMKL model provides statistically better fits to the observed spectra than the VMCFLOW model, even though the former has more free parameters than the latter. A similar result has been obtained by Pandel et al. (2005) from comprehensive analysis of DNe data by XMM-Newton. This may be attributed to the dimensional difference between galaxy clusters and DNe; the VMCFLOW model presumes spherically symmetric geometry whereas the accreting gas in DNe is basically in axial symmetry. Since the VMCFLOW model was developed for galaxy clusters, the temperature dependence of its $D E M$ can be different from that of DNe.

\subsection{Contribution of reflection component}

Fig. 5 shows the best-fit models to the XIS1 (BI-CCD) and XIS0+3 (FI-CCD) spectra of the first Suzaku observation. The intrinsic plasma emission and its reflected component are decomposed and shown in red and green curves, respectively. In $§ 3.3 .2$ we note from geometrical considerations that there must be a significant contribution to the spectra from the reflected component. As a matter of fact, a reflected continuum was detected in the Suzaku observation of SS Cyg in quiescence (Ishida et al. 2009). In VW Hyi, on the other hand, the intensity of the reflected continuum is very low, approximately $\sim 10 \%$ of the intrinsic plasma emission at $10 \mathrm{keV}$, and even weaker at lower energies. This difference stems from the fact that the maximum temperature of VW Hyi is much lower than that of SS Cyg in quiescence $(\simeq 20 \mathrm{keV})$. In the band below $\sim 10 \mathrm{keV}$, photoelectric absorption of the abundant elements dominates the opacity over Thomson scattering. Hence the reflection component is not conspicuous.

Unlike SS Cyg, the fluorescent iron emission line at $6.4 \mathrm{keV}$ is almost invisible in VW Hyi (Fig. 3). This can also be attributed to the lower maximum temperature of the continuum. The continuum X-ray photons do not have enough energy to ionize K-shell electrons of iron, whose binding energy is $7.1 \mathrm{keV}$.

\subsection{Mass accretion rate and its uncertainty}

In $\S 3.5$ we have summarized the mass accretion rates during the Suzaku and XMM-Newton observations (table 5). The mass accretion rates calculated based on the bolometric fluxes are in the range $54-70 \%$ of the best-fit $\dot{M}$ of the VMCFLOW model. Possible reasons for this inconsistency are as follows. First, as described in $\S 4.1$, the model parameter $\dot{M}$ in VMCVLOw, which is based on eq. (3) assumes a $3 \mathrm{D}$ cooling flow geometry whereas the boundary layer is rather close to $2 \mathrm{D}$. This geometrical difference possibly affects the cooling efficiency of the plasma. As a matter of fact, the CEVMKL model, which can adjust the temperature dependence of $D E M$, provides a better fit to the spectra (§3.4). Second, there remains a significant uncertainty in the white dwarf mass $M_{\mathrm{WD}}=0.63 \pm 0.15 M_{\odot}$ (Schoembs \& Vogt 1981). This can, together with the associated $R_{\mathrm{WD}}$ uncertainty, result in some $50 \%$ uncertainty for $\dot{M}$ calculated from the 
Table 5. Mass accretion rates in the unit of $10^{-12} M_{\odot} \mathrm{yr}^{-1}$ obtained from the spectral fits.

\begin{tabular}{llcccc}
\hline Model & Source & 406009020 & 406009030 & 406009040 & 0111970301 \\
\hline \multirow{2}{*}{ VMCVLOW } & model parameter & $2.45_{-0.05}^{+0.07}$ & $1.92_{-0.06}^{+0.05}$ & $2.02_{-0.05}^{+0.05}$ & $1.40_{-0.03}^{+0.03}$ \\
& bolometric flux & $1.67_{-0.05}^{+0.03}$ & $1.35_{-0.03}^{+0.04}$ & $1.39_{-0.03}^{+0.03}$ & $0.86_{-0.02}^{+0.03}$ \\
\multirow{2}{*}{ CEVMKL } & bolometric flux & $1.58_{-0.05}^{+0.03}$ & $1.30_{-0.03}^{+0.04}$ & $1.34_{-0.03}^{+0.03}$ & $0.76_{-0.02}^{+0.03}$ \\
\hline
\end{tabular}

\section{0} $\mathrm{BI}-\mathrm{CCD}$

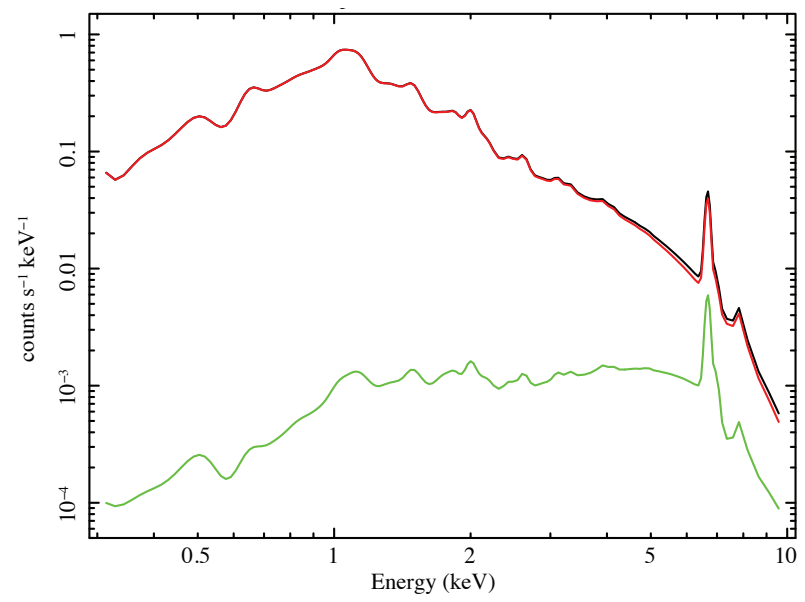

FI-CCD

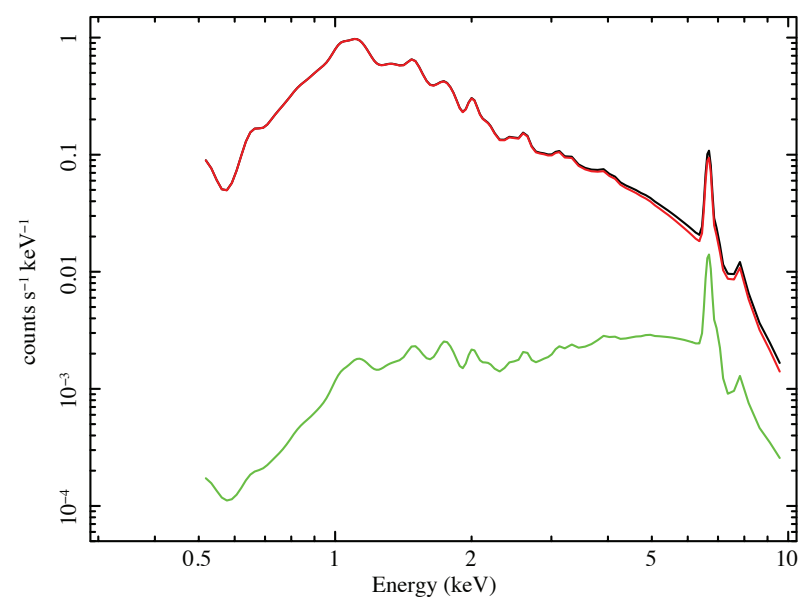

Figure 5. An example spectral fit to the first Suzaku observation with the CEVMKL model being multiplied with REFLECT, demonstrating contribution of the reflection component with the green curve. Black and red curves show models of the total spectrum and the intrinsic plasma emission spectrum, respectively.

fluxes. Finally, it is possible that the white dwarf rotates at a non-negligibly high speed compared to the Keplerian velocity at the white dwarf surface. When the accreting matter arrives at the boundary layer, it retains kinetic energy of $\left(R_{\mathrm{WD}} \omega_{\mathrm{K}}\right)^{2} / 2$ per unit mass, where $\omega_{\mathrm{K}}$ is the Keplerian angular velocity on the white dwarf surface in the equatorial plane. Here we neglect the geometrical extent of the boundary layer for simplicity. If the white dwarf does not rotate at all, all this energy should be released in the boundary layer for the matter to settle onto the white dwarf. If, on the other hand, the white dwarf rotates at a finite angular velocity $\omega$, the matter is able to accrete onto the white dwarf by releasing the energy

$\frac{1}{2} R_{\mathrm{WD}}^{2}\left(\omega_{\mathrm{K}}^{2}-\omega^{2}\right)=\frac{1}{2} \frac{G M_{\mathrm{WD}}}{R_{\mathrm{WD}}}\left[1-\left(\frac{\omega}{\omega_{\mathrm{K}}}\right)^{2}\right]$

per unit mass. Consequently, the factor $1 / 2$ in eq. (3) should be replaced by $(1 / 2)\left[1-\left(\omega / \omega_{\mathrm{K}}\right)^{2}\right]$. The systematic differences apparent in table 5 can probably be attributed to these uncertainties and errors.

\subsection{Evolution of the mass accretion rate \\ 4.4.1 Evolution of $\dot{M}$ with supercycle phase}

In Fig. 6 we show a time history of the mass accretion rate evaluated in $\S 3.4$ and $\S 4.3$ in comparison to the $\mathrm{V}$-magnitude light curves drawn since the onset of the last superoutburst before the Suzaku and XMM-Newton observations. Here we plot the best-fit parameter $\dot{M}$ of VMCFLOW listed in table 3 . Here we have added a few more data points from past observations. Of the two $A S C A$ points, the first one comes from the observation carried out in 1993 November, which is presented in Baskill et al. (2005). We have reanalysed the data to find that our bolometric flux coincides with theirs within $10 \%$. The other is taken from the observation in 1995 March, which has never been published. We have processed the data in the same way as the first ASCA observation, and have found that VW Hyi was extremely faint. We plot the best-fit $\dot{M}$ of the VMCFLOW model for both $A S C A$ datasets. The third data point originates from the Ginga and ROSAT observation which is published by Wheatley et al. (1996). The authors of this paper fitted the contemporaneous Ginga and ROSAT spectra with a two component thermal bremsstrahlung model. We have calculated the bolometric flux from their best-fit model parameters, and derived the mass accretion rate through eq. (3). In converting this to the VMCFLOW parameter $\dot{M}$, we refer to the conversion factor $54 \%-70 \%$ from the VMCFLOW parameter $\dot{M}$ to the flux-based $\dot{M}(\S 4.3)$. We would like to remark, however, that this Ginga+ROSAT observation was carried out only a few days after the onset of an outburst, and the hard X-ray counting rate had been increasing systematically (see Fig. 1 of Wheatley et al. 1996). This implies that the boundary layer had not reached a steady state.

At first sight of the four data points from the Suzaku and $X M M$-Newton observations, there seems a steady decline of the mass accretion rate as time passes. Although the first Suzaku observation has the highest mass accretion rate of the four, the second Suzaku observation gives a similar mass accretion rate as the third observation, which was carried 


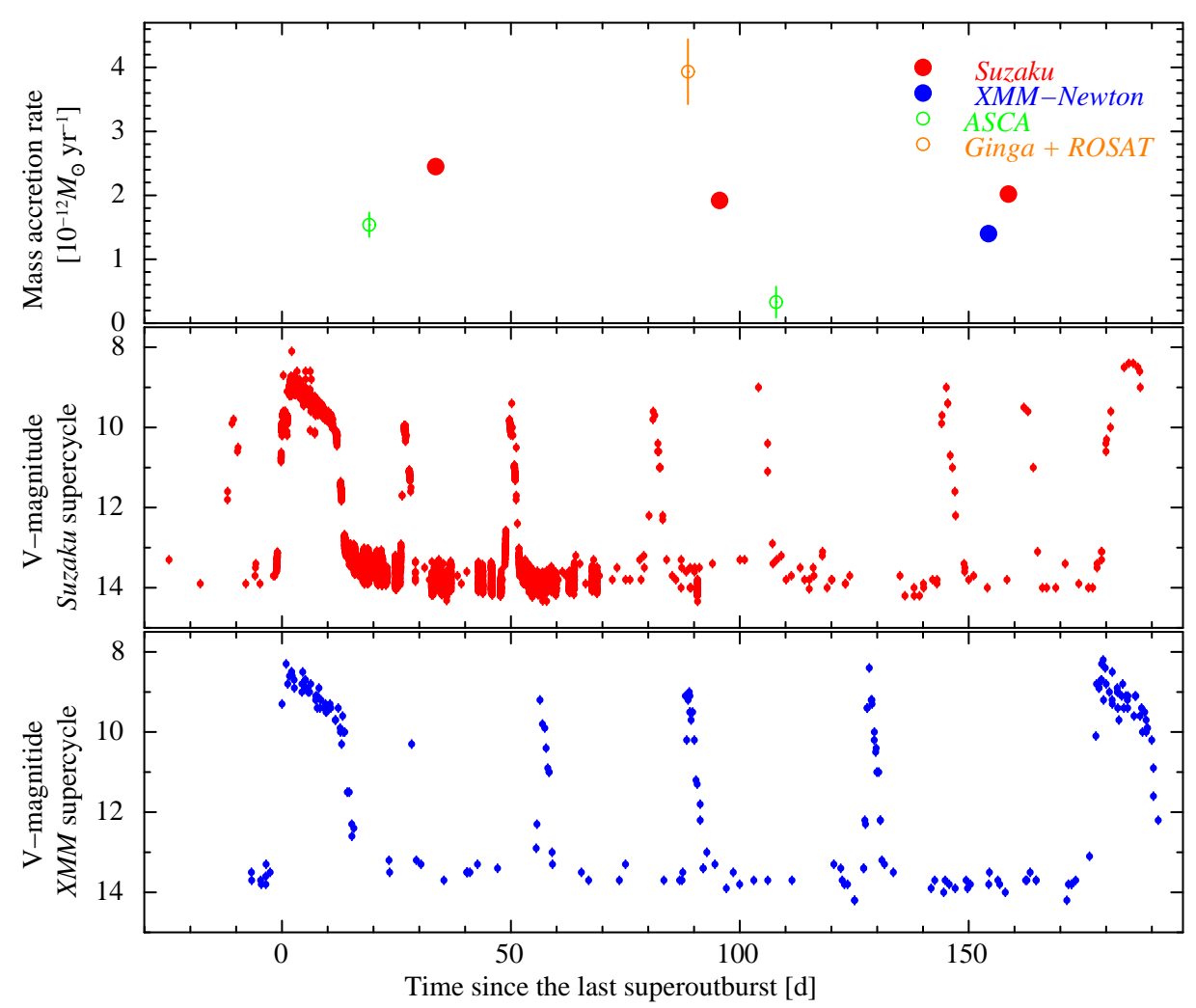

Figure 6. Time history of the mass accretion rate, the VMCFLOW parameter $\dot{M}$, since the last superoutburst. The filled circles coloured with red and blue in the top panel are from Suzaku and XMM-Newton, respectively. Open circles coloured with green and orange are from ASCA observations (Baskill et al. 2005) and contemporaneous Ginga+ROSAT observations (Wheatley et al. 1996), respectively. The middle and the bottom panels show the V-magnitude light curve corresponding to the same supercycles of the Suzaku and XMMNewton observations (the same as those in Fig. 1 and 2).

out some 60 days after the second. Moreover, the mass accretion rate from the $X M M-N$ ewton observation, which was performed at nearly the same epoch as the third Suzaku observation, is smaller than that of the third Suzaku observation by $\sim 30 \%$. As noted in $\S 3.4$, disagreement of fluxes measured with Suzaku and XMM-Newton detectors is less than 10\% (Tsujimoto et al. 2011; Ishida et al. 2011). Hence this flux disagreement is real. Although the three Suzaku observations and the XMM-Newton observation belong to different supercycles, we conclude that there is no clear trend in the mass accretion rate as a function of the time since the eruption of a superoutburst. This conclusion holds, or is even reinforced, with the $A S C A$ and Ginga + ROSAT data points being included.

We remark that, in SU UMa type DNe, the total mass of the accretion disc increases between two superoutbursts (Osaki 1996). As the disc accumulates mass, its surface density is expected to increase as time passes, and hence the mass accretion rate also increases. Such a systematic increase of the mass accretion rate is not evident from the current Suzaku and XMM-Newton observations.

\subsubsection{Evolution of $\dot{M}$ in the outburst cycle}

In Fig. 7, we have plotted the mass accretion rate as a function of the time since the last outburst (including the superoutburst). In contrast to Fig. 6, this shows a clear decreasing trend of the mass accretion rate, except for the

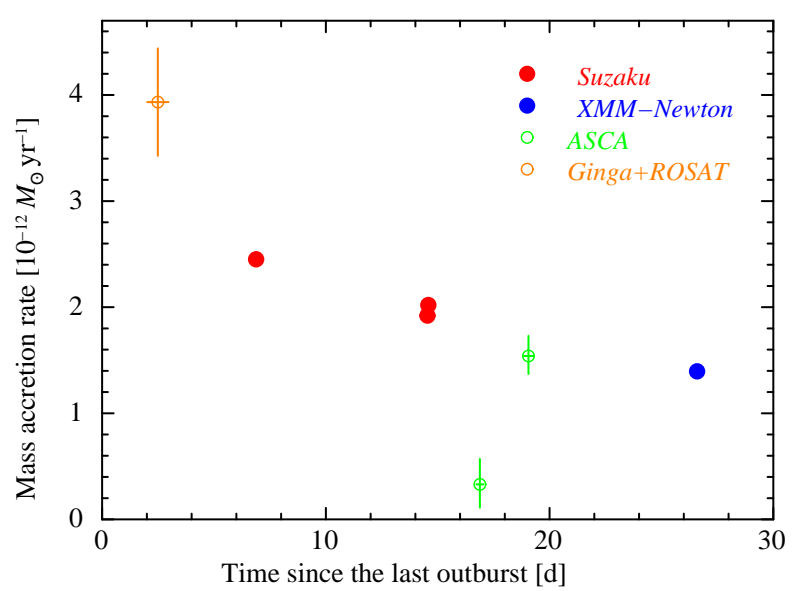

Figure 7. Evolution of the mass accretion rate as a function of the time since the last outburst. The symbols of the data points are the same as those in Fig. 6.

extremely low mass accretion rate in the second $A S C A$ observation. There are at least a couple of previous works that suggest decline of the hard X-ray flux during optical quiescence. McGowan et al. (2004) analysed four sets of RXTE PCA data of SS Cyg, each of which encompass $\sim 2$ weeks to a few months. They found that the X-ray counting rate in the band $1.3-12.2 \mathrm{keV}$ declines at a rate of $1.3 \% \mathrm{~d}^{-1}$, accumulating $40 \%$ decrease in 31 days since the instance when SS Cyg 
gets fainter than $m_{\mathrm{V}}=11.7 \mathrm{mag}$. Collins \& Wheatley $(2010)$ found that the X-ray counting rate of SU UMa in quiescence in the band 2.0-18.5 keV observed with the RXTE PCA decreases at a rate of 0.09 counts $\mathrm{s}^{-1} \mathrm{~d}^{-1}$ from 5.8 counts $\mathrm{s}^{-1}$, which implies $-1.6 \% \mathrm{~d}^{-1}$, similar to SS Cyg. The decline of the mass accretion rate in the current dataset of VW Hyi is $43 \%$ in $\sim 20 \mathrm{~d}$, implying the rate of $-2.2 \% \mathrm{~d}^{-1}$. This is of the same order as the previous two works.

The authors of these two previous works mentioned that the global decline of the mass accretion rate within the boundary layer during the quiescent phase (inter-outburst period) conflicts with the expectation of the disc instability model, which predicts increasing accretion rate during quiescence (e.g. Lasota 2001). One may invoke retardation of mass transfer through the disc. Mineshige \& Osaki (1985) showed in their disk instability simulation that the outburst is triggered by a heating wave that is initiated in the outer part of the disc and travels inward. The expected delay of an outburst in the EUV band is really detected in SS Cyg; Wheatley et al. (2003) found that the rise of the EUV flux is retarded from the onset of the optical outburst by 1.52 d. Collins \& Wheatley (2010) found that in SU UMa the hard X-ray suppression, which is expected to occur simultaneously with the EUV outburst, is retarded by $0.57 \mathrm{~d}$ on the average from the onset of the optical outburst. These results indicated that it takes $\sim 1$ day for the heating wave to reach the boundary layer from the optically bright part of the disc. Since the quiescent disc is expected to have much lower density, the time necessary for physical quantities to propagate through the disc should be much longer.

According to Fig. 1 of Wheatley et al. (1996) (and also Fig. 1 and 2), however, a normal optical outburst continues only $\sim 3$ days. The end of the outburst corresponds to the epoch when the cooling wave passes the optical disc and arrives at the entrance of the boundary layer, after which the mass accretion rate should resume as per the standard disc instability model. The increase of the hard X-ray flux (again Fig. 1 of Wheatley et al. 1996), which is direct evidence of a decline of the mass accretion rate in the boundary layer, continues at least until $\sim 6$ days after the onset of the optical outburst. This seems to conflict with the disc instability model. One may consider that Fig. 1 of Wheatley et al. (1996) did not detect the end of the outburst due to the optical flux limit, yet it is hard to believe that it takes $\sim 30 \mathrm{~d}$ (Fig. 7) for the cooling wave to arrive at the boundary layer, since $30 \mathrm{~d}$ is comparable to the average time of burst repetition (Fig. 6).

To reconcile the mass accretion rate decline in quiescence with the standard disc instability model, one may presume that the optically thick disc survives close to the orbital plane even in the optically-quiescent state, being covered with an optically thin boundary layer. Since there must be some density gradient in the disc in the direction perpendicular to the orbital plane, such a geometry may be possible. As the mass accretion rate increases, this optically thick boundary layer grows and consumes a larger fraction of the accretion energy, resulting in the apparent decline of $\dot{M}$ in the hard X-ray band. The emission from such a disc is expected to appear in the EUV band, being somewhat Comptonized by the overlying optically thin hot plasma. Such a spectral component, however, has not been detected so far; we should confess that such an optically thick component of the boundary layer in quiescence is just speculation at this moment.

On the other hand, the standard disk instability model (Lasota 2001, for example) may need to be modified. Although the model predicts an increase of the mass accretion rate through the disc in quiescence, the optical magnitude seems fairly constant throughout the quiescence phase (see Fig. 1 and 2). The optical flux originates not only from the accretion disc but also from the white dwarf and the secondary star. Of them, the emission from the white dwarf may decline during quiescence due to cooling after the outburst. It is, however, difficult to imagine that the increase of the disc emission and the decrease of the white dwarf emission just compensate each other. We also believe there is no DN that shows a steady decline of the V-magnitude (flux increase) during optical quiescence.

Finally, we briefly mention the extremely faint state of VW Hyi observed with $A S C A$ in March 1995. VW Hyi was so faint and the mass accretion rate at that time was much lower than the declining trend shown in Fig. 7. Although the spectral parameters are not constrained very well, the $A S C A$ spectrum is fitted with the VMCFLOW model with $T_{\max }=$ $11.5_{-3.1}^{+3.5} \mathrm{keV}$. The spectrum seems slightly harder than the other quiescence spectra presented in this paper. This can be attributed to a lower mass accretion rate, resulting in lower cooling efficiency of the plasma. The reason for VW Hyi to fall into such a low state should be pursued in the future.

\section{CONCLUSION}

In order to elucidate time variation of the mass accretion rate of DNe in the optically-quiescent phase, we have analysed a series of VW Hyi data taken with Suzaku. As complement, we have added one dataset from XMM-Newton. The observed spectra in the band $0.2-10 \mathrm{keV}$ are moderately well represented by multiple temperature thermal plasma emission models (VMCFLOW and CEVMKL models in XSPEC) with a maximum temperature of $5-9 \mathrm{keV}$. The bolometric luminosity is $(2.4-5.2) \times 10^{30} \mathrm{erg} \mathrm{s}^{-1}$. From the evaluation of the spectra, we have derived the mass accretion rates. According to the theoretical prediction of the disc behaviour during a supercycle of SU UMa type DNe (Osaki 1996), the disc accumulates mass throughout the entire supercycle, and the mass accretion rate through the disc is expected to increase. The measured mass accretion rate, however, does not show any clear trend with the supercycle phase. If we plot the mass accretion rate, on the other hand, against the elapsed time since the last outburst (including the superoutburst), we have found a systematic declining trend with a rate of $-2.2 \% \mathrm{~d}^{-1}$. This rate of decline is comparable to the other two DNe: SS Cyg $\left(-1.3 \% \mathrm{~d}^{-1}\right.$, McGowan et al. 2004) and SU UMa $\left(-1.6 \% \mathrm{~d}^{-1}\right.$, Collins \& Wheatley 2010). This trend holds, or is even reinforced by adding a few datasets from Ginga, ROSAT and ASCA. The decrease of the mass accretion rate through the boundary layer during the opticallyquiescent state is, however, hard to reconcile with the standard disc instability model (e.g. Lasota 2001) in which the mass accretion rate is predicted to increase throughout the quiescence phase. This discrepancy may be resolved if one may speculate that an optically thick disc survives in the orbital plane of the boundary layer even during the optically- 
quiescent state, consuming a larger fraction of the accretion energy than the optically thin hot plasma as the mass accretion rate increases. Alternatively, the standard disc instability model may need to be modified. We obviously need further observation and theoretical consideration to finally resolve this discrepancy.

\section{ACKNOWLEDGEMENTS}

The authors are grateful to the anonymous referee for useful suggestions and comments. They would like to express special gratitude to Dr. Koji Mukai for his useful information and constructive discussion. They are also grateful to Prof. Takaya Ohashi and Dr. Magnus Axelsson for his invaluable comments to the initial draft of this paper.

This work has made use of data from the European Space Agency (ESA) mission Gaia $^{4}$, processed by the Gaia Data Processing and Analysis Consortium (DPAC) ${ }^{5}$. Funding for the DPAC has been provided by national institutions, in particular the institutions participating in the Gaia Multilateral Agreement.

\section{REFERENCES}

Anders E., Grevesse N., 1989, Geochimica Cosmochimica Acta, 53,197

Arnaud K. A., 1996, in Jacoby G. H., Barnes J., eds, Astronomical Society of the Pacific Conference Series Vol. 101, Astronomical Data Analysis Software and Systems V. p. 17

Barrett P., 1996, PASP, 108, 412

Baskill D. S., Wheatley P. J., Osborne J. P., 2005, MNRAS, 357,626

Collins D. J., Wheatley P. J., 2010, MNRAS, 402, 1816

Fabian A. C., 1994, ARA\&A, 32, 277

Gaia Collaboration et al., 2016, A\&A, 595, A1

Gaia Collaboration et al., 2018, A\&A, 616, A1

Gehrels N., Williams E. D., 1993, ApJ, 418, L25

Hōshi R., 1979, Progress of Theoretical Physics, 61, 1307

Hirose M., Osaki Y., 1990, PASJ, 42, 135

Ishida M., Okada S., Hayashi T., Nakamura R., Terada Y., Mukai K., Hamaguchi K., 2009, PASJ, 61, S77

Ishida M., et al., 2011, PASJ, 63, S657

Jansen F., et al., 2001, A\&A, 365, L1

Kokubun M., et al., 2007, PASJ, 59, 53

Koyama K., et al., 2007, PASJ, 59, 23

Lasota J.-P., 2001, New Astron. Rev., 45, 449

Magdziarz P., Zdziarski A. A., 1995, MNRAS, 273, 837

McGowan K. E., Priedhorsky W. C., Trudolyubov S. P., 2004, ApJ, 601, 1100

Meyer F., Meyer-Hofmeister E., 1981, A\&A, 104, L10

Mineshige S., Osaki Y., 1985, PASJ, 37, 1

Mitsuda K., et al., 2007, PASJ, 59, S1

Mukai K., Wood J. H., Naylor T., Schlegel E. M., Swank J. H., 1997, ApJ, 475, 812

Mushotzky R. F., Szymkowiak A. E., 1988, in Fabian A. C., ed., NATO Advanced Science Institutes (ASI) Series C Vol. 229, NATO Advanced Science Institutes (ASI) Series C. pp 53-62, doi:10.1007/978-94-009-2953-1_6

Nauenberg M., 1972, ApJ, 175, 417

Osaki Y., 1974, PASJ, 26, 429

4 https://www. cosmos.esa.int/gaia

5 https://www.cosmos.esa.int/web/gaia/dpac/consortium
Osaki Y., 1989, PASJ, 41, 1005

Osaki Y., 1996, PASP, 108, 39

Pandel D., Córdova F. A., Howell S. B., 2003, MNRAS, 346, 1231

Pandel D., Córdova F. A., Mason K. O., Priedhorsky W. C., 2005, ApJ, 626, 396

Patterson J., Raymond J. C., 1985, ApJ, 292, 535

Polidan R. S., Mauche C. W., Wade R. A., 1990, ApJ, 356, 211

Pringle J. E., Savonije G. J., 1979, MNRAS, 187, 777

Ritter H., Kolb U., 2003, A\&A, 404, 301

Schoembs R., Vogt N., 1981, A\&A, 97, 185

Serlemitsos P. J., et al., 2007, PASJ, 59, S9

Takahashi T., et al., 2007, PASJ, 59, 35

Tsujimoto M., et al., 2011, A\&A, 525, A25

Wada Q., Tsujimoto M., Ebisawa K., Hayashi T., 2017, PASJ, 69,10

Warner B., 1987, MNRAS, 227, 23

Warner B., 2003, Cataclysmic Variable Stars, doi:10.1017/CBO9780511586491.

Wheatley P. J., Verbunt F., Belloni T., Watson M. G., Naylor T., Ishida M., Duck S. R., Pfeffermann E., 1996, A\&A, 307, 137

Wheatley P. J., Mauche C. W., Mattei J. A., 2003, MNRAS, 345,49

Whitehurst R., 1988, MNRAS, 232, 35

van Teeseling A., Beuermann K., Verbunt F., 1996, A\&A, 315,467

This paper has been typeset from a $\mathrm{T}_{\mathrm{E}} \mathrm{X} / \mathrm{LAT} \mathrm{TX}$ file prepared by the author. 